\title{
The Effect of Allocative Efficiency of Free Markets on Entropy and its Implications on Taxes
}

\author{
By Stephan Unger*
}

\begin{abstract}
This article shows that the entropy in a free market is maximized under the allocative efficiency condition. In contrast to that, it is shown that any pre-determined allocation, as it is the case with the collection and distribution of taxes, exhibits a higher probability of minimizing the entropy in the system, where the loss in entropy corresponds to the deadweight loss caused by the excess burden of taxation. The implications are that any chaotic system, or exchange economy, converges to an optimal structure of wealth distribution which maximizes social welfare, in contrast to a randomly, pre-determined distribution of wealth.
\end{abstract}

Keywords: Entropy, Social Welfare, Deadweight Loss, Perfect Competition, Allocative Efficiency, Taxes.

\section{Introduction}

Adam Smith argued in his seminal work, "The Wealth of Nations" (1776), that the invisible hand of free markets, also known as the first welfare theorem, channels an economic system to reach the correctly required level of production. This required level of production would lead to a system where all resources are optimal allocated.

Arrow and Debreu showed mathematically that free markets reach an equilibrium that is Pareto efficient in allocation (Frank 2003). In the state of a Pareto efficient allocation the entropy of the system is at its maximum.

The notion of entropy was first defined by Carnot in 1803 (see Oliveira 2014) but got adapted by Boltzmann (1866), Gibbs (1878) and Maxwell (1871). The domain of entropy is widely spread among thermodynamics, statistical mechanics, communications and information theory.

Technically, entropy is an extensive state variable that is definable for any material substance or any system. The term "extensive" means that it is proportional to the "size" of the system (like volume or mass) in contrast to an "intensive" variable (like temperature, pressure or density). The term, along with the underlying concept, was introduced by Rudolph Clausius, in the $19^{\text {th }}$ century, to help explain the tendency of temperature, pressure, density and chemical gradients (in fact, all sorts of gradients) to flatten out and gradually disappear over time. The physical law behind the concept is deceptively simple to state: If the system is isolated and closed, so that it does not exchange matter or energy with any other system, its entropy increases with every physical action or transformation that occurs inside the system.

*Assistant Professor, Saint Anselm College, USA. 
Entropy can never decrease in an isolated system or in the universe as a whole. When the isolated system reaches a state of internal equilibrium its entropy is maximized. When two systems interact with each other, their total combined entropy also tends to increase over time. This non-decreasing property, roughly speaking, is known as the Second Law of Thermodynamics, or just the "entropy law". (Ayres 1996)

In information theory, entropy defines the average amount of information produced by a system. In terms of an exchange economy, entropy encompasses all information about all transactions taking place. Considering that any kind of wealth distribution among all agents in this system is based on this information, a state of maximum entropy is desirable. Jaynes (1965) established the principle of maximum entropy which states that "the probability distribution which best represents the current state of knowledge is the one with largest entropy".

The distribution of wealth in a system in which entropy is not maximized means that the distribution does not consider all information embedded in the system, meaning all economic data and variables. Therefore the realized distribution can also not be optimal. According to the second law of thermodynamics, every natural system tends to maximize entropy by increasing its complexity. By adding information to the system, the complexity of the system increases. By sufficient addition of information it can be shown that the system converges to an equilibrium which is optimal.

Such collective mechanisms are e.g. given in a free market environment where each unrestricted transaction taking place serves as an additional input of information, thus optimizing the distribution of wealth in an economy.

There are two ways to determine the collection and distribution of wealth in an economy: Through a social market economy or a free market. The existence of a centralized entity in a social market economy is sufficient but not necessary as it might administer the actual distribution of wealth. Concurrently it is highly inefficient to have the centralized entity decide about the shape of wealth distribution as the probability that this determination is optimal converges to zero with increasing amounts of trials to realize an optimal distribution of wealth.

In contrast to that, a determination of wealth by a free market is based on the system's agent's learning progress through trial and error by the implicit generation of information in every iteration. Robinson (1969) suggests that the seller may be conceived to equate marginal revenue to marginal cost "either by estimating the demand price and the cost of various outputs, or by a process of trial and error".

Therefore learning outcome is evaluated at each distribution step in terms of realized entropy. The probability that the system reaches an optimal status, with respect to its maximum entropy, increases as the number of trials increases as information $N \rightarrow \infty$. This paper shows how the maximization of entropy leads to an optimal distribution of wealth.

I will first give an overview of wealth distribution in a social market environment vs. a free market environment. Then we take a look at the starting conditions necessary to determine the evolution of a tax collection regime with subsequent redistribution of wealth vs. the consolidation of a wealth distribution in a free market. 
After explaining the difference of wealth distribution evolution in both systems, I will explain the concept of entropy maximization in an exchange economy, showing that the quality of a certain realized wealth distribution can be measured by the Kullback-Leibler divergence, even if the optimal distribution is unknown.

Finally, I will analyze centralized taxation and its implications on the deadweight loss, as well as its meaning to entropy in an exchange system. Additionally I will briefly explain the effect of entropy on monopolies.

\section{Wealth Distribution in a Social Market vs. Free Market}

"Prior to the passage of the $16^{\text {th }}$ Amendment in 1913, the United States government funded its operations mainly through excise taxes, tariffs, customs duties and public land sales. The federal government had relatively few expenses compared to today and did not have as much need to raise large amounts of money. The U.S. government was capable of paying for its expenses without an income tax prior to 1913 largely because it had fewer responsibilities. Thomas Eddlem noted in "The New American" that the federal government's responsibilities were limited to basic operational matters and did not include such modern expenses as social insurance programs, welfare programs or agricultural subsidies." (Gabriel 2018)

Article I, Section 9 of the U.S. Constitution states, "No Capitation, or other direct, Tax shall be laid, unless in Proportion to the Census or enumeration herein before directed to be taken." A federal income tax had been enacted in 1861, but was struck down as unconstitutional by the Supreme Court in 1895 because it was found to be a direct tax outside the constitutional constraints. Congress removed these limits in 1913 with the passage of the 16th Amendment, which allows it to impose income taxes specifically, "without regard to any census or enumeration."

The history shows that a social system is able to function without the imposition of personal income taxes. Raising taxes on income lowers purchasing power and the allocation ability. If no taxes were imposed, an agent could determine his own allocation and spending amount towards system relevant factors such as energy, health, social security or pensions.

On the supply side, every agent would observe the realized spending distribution and prices would be set according to the willingness of providing the services requested. The probability that both agents don't equilibrate is initially very high, thus information entropy is very low in this system, because none of the agents knows the other agents adjustment capability.

Negotiation among both agents might lead to a market clearing price. This price might be an exchange price for two commodities, but might also be the exchange between one unit of labor and a commodity. For the system, information entropy would increase. Considering many agents, entropy is very small at this stage with just two agents contributing towards an n-agent's system entropy with just one cleared market price.

The process of finding a market equilibrium in which some services clear will accelerate as soon as more and more information is revealed on the supply as well 
as on the demand side, thus entropy increases as the complexity increases, because more and more transaction information has to be considered. As entropy increases, resources are allocated more and more efficient towards relevant and market clearing sectors. All sectors start getting priced fairly which means that wealth starts getting distributed optimally. Moreover, economies of scale will lead to fair pricing, e.g. electricity provider will be able to produce at rates which consumers will be willing to accept.

For a market clearing system with maximum entropy, the probability that the wealth is distributed optimally converges to 1 . Therefore, a system which predetermines the shape of the distribution of the resources just realizes one possible outcome of an N-trial entropy-maximizing system. The probability that this outcome is optimal vanishes with increasing number of trials.

\section{Prerequisites}

\section{Social Market System}

First we need to define the starting conditions for the two systems subject to comparison: The social market exchange economy and the free market economy. In order to run an N-trial exchange system the starting values are set randomly in both systems.

For the social market economy we can assume that a central entity chooses with a certain probability the distribution of wealth $\mathrm{W}$, conditional on the tax collection distribution $\mathrm{T}$, based on available market information such that

$$
P(W \mid T)=\frac{P(T \mid W) P(W)}{P(T)},
$$

provided that $P(W), P(T)>0 . P(T \mid W)$ is hard to evaluate since in a social market environment, the distribution collection of taxes given a certain wealth distribution is exposed and subject to political debate and choice. Therefore we can interpret $P(W \mid T)$ as measure for redistribution of wealth. $P(W)$ is the probability of realization of a certain wealth distribution and $P(T)$ is the probability of a certain tax collection choice.

The tax brackets are subject to change, based on constant updates. The problem a social market system faces is that with increasing number of updates (or iterations), the complexity of the system increases too. This makes it impossible for a central entity, besides political pressure, to update its distribution conditional on all information.

Moreover, the social market system faces this problem in two ways: First, in the determination of the tax collection brackets, and second in the choice of redistribution of the wealth. As we will see, the problem of system complexity turns out to be crucial when it comes to the determination of a socially optimal distribution of wealth. 


\section{Free Market System}

A fundamental requirement for a free market to exist is pure competition. There is general agreement upon concrete circumstances which are prerequisite to the functioning of pure competition. Professor Chamberlin asks: (1) that the number of buyers and sellers be large "so that the influence of any one or several in combination is negligible"; (2) and that an identical good be offered by all the sellers. (Triffin 1947c)

Perfection of the market is identified with the identity of the product. In a free market environment the starting condition is set by buyers and sellers who negotiate about a transaction price. Their negotiation may also be based on given market information, e.g. cost of resource, cost of labor, comparable goods, etc. By realization of transaction prices $A$, the distribution $\mathrm{W}$ is formed successively, where each new transaction price, displays new information, serving as input for the generation of subsequent transaction prices. Thus, the distribution of wealth in a free market environment is based on realized transaction prices:

$$
P(W \mid A(n))=\frac{P(A \mid W) P(W)}{P(A)},
$$

provided that $P(W), P(A)>0 . P(A \mid W)$ is generated successively, simply by realization of transaction prices based on the given distribution of wealth, where the starting distribution of wealth might not be equal among the agents due to different endowments of resources, etc.

Since $\mathrm{T}$ and $\mathrm{A}$ are functions of $\mathrm{N}$ iteration steps, both systems might face the same starting conditions, but as we will see, will develop in a different way over time, due to different complexity accumulation.

\section{Entropy Maximization in an Exchange Economy}

Assuming that we denote the unknown optimal distribution of wealth by $\mathrm{X}$ and the approximating distribution, either set by a government, as $Y_{G}$ or determined by the free market, as $Y_{F}$. To determine which distribution approximates better $\mathrm{X}$, we can measure the Kullback-Leibler (KL) divergence. Since the distribution with the least distance to the real distribution is the preferable one, we need to minimize the KL-divergence. By minimizing the KL-divergence between $\mathrm{X}$ and $Y_{G}$, respectively $\mathrm{X}$ and $Y_{F}$ we automatically maximize the entropy of the system as

$$
\begin{aligned}
& \tilde{g}(x)_{N} \rightarrow p(x) \Rightarrow D\left(X \| Y_{G}\right)=-\sum_{x=1}^{n} p(x) \log \left(\frac{g(x)}{p(x)}\right) \rightarrow 0 \text { as } N \rightarrow \infty, \\
& \tilde{f}(x)_{N} \rightarrow p(x) \Rightarrow D\left(X \| Y_{F}\right)=-\sum_{x=1}^{n} p(x) \log \left(\frac{f(x)}{p(x)}\right) \rightarrow 0 \text { as } N \rightarrow \infty,
\end{aligned}
$$


where $\mathrm{X}$ is distributed as $p(x), Y_{G}$ as $\mathrm{g}(\mathrm{x})$ and $Y_{F}$ as $\mathrm{f}(\mathrm{x})$. For large $\mathrm{N}$ we can find the best parameters, which minimize the KL-divergence for both approximating distributions:

$D\left(X \| Y_{G}\right)=-\sum_{x=1}^{M} p(x) \log \left(\frac{g(x ; w)}{p(x)}\right)=-\sum_{x=1}^{M} p(x) \log (\mathrm{p}(\mathrm{x}))$
$-\sum_{x=1}^{M} p(x) \log (\mathrm{g}(\mathrm{x} ; \mathrm{w}))$,

$D\left(X \| Y_{F}\right)=-\sum_{x=1}^{M} p(x) \log \left(\frac{f(x ; w)}{p(x)}\right)=-\sum_{x=1}^{M} p(x) \log (\mathrm{p}(\mathrm{x}))$

$-\sum_{x=1}^{M} p(x) \log (\mathrm{f}(\mathrm{x} ; \mathrm{w}))$,

where $-\sum_{x=1}^{M} p(x) \log (\mathrm{p}(\mathrm{x}))$ is constant.

Therefore the KL-divergence gets minimal if the terms $-\sum_{x=1}^{M} p(x) \log (\mathrm{g}(\mathrm{x} ; \mathrm{w}))$ and $-\sum_{x=1}^{M} p(x) \log (\mathrm{f}(\mathrm{x} ; \mathrm{w}))$, defining the entropy, get maximized. That means that with increasing number of $\mathrm{N}$ transaction prices, the KL-divergence converges to its $\min$.

Thus, a free market environment can always generate and encompass more information than any fixed chosen distribution. This leads to the conclusion that a free market framework approximates more accurate any optimal distribution of wealth than a chosen distribution. The reason lies in the fact that any central entity which chooses a certain distribution can only consider a certain amount of complexity of the system, and is thus limited in gathering all market information. Of course this condition holds just if the rate at which the complexity of the system increases is below the rate at which information is generated.

\section{Efficient Allocation}

To prevent a system without tax payments from a collapse due to social unrest the agents will have to self-organize by efficient allocation of their resources. Since the system consists of learning agents, the system will yield for an equilibrium $i^{*}$ with probability $p_{i^{*}}$, which is Pareto-optimal for all system entities.

For production to happen at marginal cost and equalizing marginal benefit of the consumers, all information has to be known (meaning that the system complexity is at its maximum), thus entropy has to be maximized.

In order to ensure that the system is allocating its resources efficiently by maximizing its entropy it has to satisfy following Shore and Johnson axioms:

- The maximum $p_{i^{*}}$ has to be unique.

- Predictions about the resource allocation should be coordinate invariant.

- Subset independence: If the probability or realization of a resource allocation path $p_{k^{*}}$ is increased and the probability or realization of 
another resource allocation path $p_{j^{*}}$ is decreased then no other realizations $p_{n^{*}}$ are affected.

- System independence: The predicted joint probability of realizing two resource allocations must be the product of the predicted marginal probabilities.

Efficient allocation prevails when the marginal cost equal marginal revenue, thus when the quantities $Q^{*}$ are exchanged at an efficient price $P^{*}$. Both functions, $\mathrm{g}(\mathrm{x})$ and $\mathrm{f}(\mathrm{x})$, try to approximate these efficient quantities and prices.

In order to measure if $\mathrm{g}(\mathrm{x})$ or $\mathrm{f}(\mathrm{x})$ better approximates $\mathrm{X}$, we have to take the model which maximizes the likelihood function $\log (L)=\sum_{x=1}^{M} \tilde{p}(x) \log (\mathrm{p}(\mathrm{x} ; \mathrm{w}))$. By using the Bayesian Information Criterion (BIC) we can determine which distribution approximates better the optimal distribution:

$$
B I C=-2 \log (L)+K * \log (n) .
$$

A small BIC is preferred, therefore we are interested in evaluating the performance of $\mathrm{g}(\mathrm{x})$ and $\mathrm{f}(\mathrm{x})$ when $N \rightarrow \infty$ and how the BIC changes:

$$
\begin{gathered}
\frac{d B I C}{d n}=K * \frac{d}{d n}(\log (n)) * \frac{d}{d n} \log (n), \\
\frac{d B I C}{d n}=\frac{K}{n}
\end{gathered}
$$

We can see that the rate at which the BIC information criterion gets minimal is when $N \rightarrow \infty$. Since $\mathrm{g}(\mathrm{x})$ is fixed, $N<\infty$ whereas $\mathrm{f}(\mathrm{x})$ is not fixed, thus allows $N \rightarrow \infty$.

The rate at which the BIC approximately changes is:

$$
\begin{gathered}
P_{1}+\left(\frac{K}{n}\right)\left(Q^{*}-\hat{Q}\right)=P_{2}+\left(\frac{K}{n}\right)\left(Q^{*}-\hat{Q}\right), \\
\left(P_{2}-P_{1}\right)=2 * \frac{K}{n}\left(Q^{*}-\hat{Q}\right), \\
\frac{K}{n}=\frac{1}{2} * \frac{\left(P_{2}-P_{1}\right)}{\left(Q^{*}-\hat{Q}\right)}, \\
d\left(P^{*}, \widehat{P}\right) \wedge\left(Q^{*}-\hat{Q}\right)=\left\|\frac{K}{n}\right\|=\left\|-\frac{1}{2} *\left[\log \left(P_{2}-P_{1}\right)+\log \left(Q^{*}-\hat{Q}\right)\right]\right\| .
\end{gathered}
$$

A system without a central entity determining how many resources are levied and subsequently allocated is automatically not able to maximize its entropy.

In such a system, the entropy $H(X)$ is the average information available to all entities which are part of the system:

$$
H(X)=-\sum_{p_{i}} p_{i} \log _{2}\left(p_{i}\right) .
$$


In a system where a central entity determines the resource allocation, the resource allocation is a deterministic function, or in other words, the probability for a random allocation to a certain project to be optimal is zero:

If $p_{i}=0$, then $p_{i} \log _{2}\left(p_{i}\right)=0$ since

$$
\lim _{p \rightarrow 0} p_{i} \log _{2}\left(p_{i}\right)=0 \text {. }
$$

The result implies that the probability for an infinite set of trials to minimize the entropy of the exchange system is higher than for just one pre-determined distribution of allocable resources.

This implies that resource allocation is only efficient in free markets.

Any pre-determined resource allocation is just one possible outcome of any randomly generated resource allocation. Equipping a central agency with the power to impose and levy taxes means adding constraints to the system. If every entity in the system is free to choose its optimal resource allocation with respect to its individual risk preference and utility function, then the aggregate utility is Pareto-optimal. This implies also that such a system would be socially sustainable.

\section{Deadweight Loss}

The deadweight loss (DWL) is defined as the loss in welfare by realization of an equilibrium in an exchange economy under imposed taxes. The socially optimal equilibrium should prevail where marginal cost of production equal the marginal cost of benefit, or when supply meets demand. By the imposition of taxes, the market is just able to realize quantities less than it could produce in the optimum and faces a reduction in the marginal benefit as realized prices for demand side are higher and on the supply side are lower than in the social optimum. The implications of this loss in welfare are well known (Hausman 1981, Case 1999, Hines 1999, Lind and Granqvist 2010). What is interesting is how the rate of complexity reduction, and thus the loss in entropy in a system with a fixed choice of wealth distribution, can explain the size of the deadweight loss:

$$
\begin{gathered}
D W L=\frac{1}{2}\left(P_{2}-P^{*}\right)\left(Q^{*}-\widehat{Q}\right)+\frac{1}{2}\left(P^{*}-P_{2}\right)\left(Q^{*}-\hat{Q}\right) \\
=\frac{1}{2}\left(Q^{*}-\hat{Q}\right)\left[\left(P_{2}-P^{*}\right)+\left(P^{*}-P_{1}\right)\right]=\frac{1}{2}\left(Q^{*}-\hat{Q}\right)\left(P_{2}-P_{1}\right), \\
D W L=d\left(P^{*}, \hat{P}\right) \wedge\left(Q^{*}-\widehat{Q}\right)=\left\|\frac{K}{n}\right\| \\
=\left\|\frac{1}{2} *\left[\log \left(P_{2}-P_{1}\right)+\log \left(Q^{*}-\hat{Q}\right)\right]\right\|, \\
\Rightarrow D W L=\frac{d(B I C)}{d n} *
\end{gathered}
$$


The welfare loss caused by taxation can therefore be explained by the rate at which information cannot further contribute towards the complexity of the system caused by a determined tax brackets and wealth distribution. The difference between a social market entropy and a free market entropy could be interpreted as the opportunity costs of information.

An increase in information, as given in a free markets setup, maximizes the complexity, thus the entropy of the system, and yields to an optimal wealth distribution. But since the complexity of the system increases not to the power of $n$ (the number of information bits), the BIC information criterion tends to get minimal as $N \rightarrow \infty$, where the BIC information criterion measures the KullbackLeibler (KL) divergence between an unknown social optimal wealth distribution and a realized wealth distribution. The reason for the superiority of free markets vs. a pre-determined wealth distribution can be explained by the phenomenon that the increase in information entropy of the system leads to allocative efficiency in the market, which minimizes the KL-divergence, as can be shown and measured by the BIC.

The rate at which the optimal wealth distribution is approximated, respectively the pre-determined wealth distribution is outperformed, corresponds to the rate at which information is added to the system, namely

$$
H(X)=-\sum_{i} p_{i} \sum_{x=1}^{n} p(x) \log (x) \propto \frac{d B I C}{d n},
$$

where $\mathrm{i}$ is a welfare-state and $p_{i}(x)$ is the probability of $\mathrm{x}$ given $\mathrm{i}$ as the previous welfare-state with a given wealth distribution.

\section{Allocation under a Monopoly}

One of the critics of free markets is that they tend to form monopolies that allocate resources non-optimally. Monopoly exists for Mises (1963) when "...the whole supply of the commodity is controlled by a single seller or a group of sellers acting in concert". He further states that if monopoly prices do exist, then they are an "infringement of the supremacy of the consumers and the democracy of the market". Mises also argues that although most monopolies and monopoly prices are made possible by government intervention in the free market (tariffs, licenses, etc.), there are certain instances in which monopoly (and monopoly prices) arise in the unhampered market. He specifically mentions natural resource monopoly, geographic monopoly, limited-space monopoly, and monopoly that might arise because consumers place a "special confidence...on the individual or firm concerned on account of previous experience," as with certain trademarked drugs.

But for Rothbard (1962) there is no social "problem" associated with monopoly in a free market. Monopoly prices cannot be defined logically, let alone established in a free market. According to Kirzner (1973), if markets are always competitive so long as there is freedom to buy and sell, then in a free market there is always competition and never any monopoly. 
Paretian analysis defines competition as incomplete when marginal costs are increasing and as complete when they are decreasing. When costs are decreasing the rules of profit maximization induce the firm, not to limit its production at a certain level, but to expand it indefinitely. In the opposite case, when increasing marginal costs limit the size of the profit maximizing output, Pareto considers that competition is "incomplete": Each producer finds it advantageous to limit his supply. (Triffin 1947a)

In the case of $N \rightarrow \infty$, under independent trials, we can assume that provided information leads to an efficient usage of resources and therefore to cost reduction in a long term. Relating the existence of monopolies to the reduction of entropy in a system we can measure the loss of entropy again at the same rate as the loss of entropy in the case of the dead weight loss.

An interesting point arises when we consider interdependence in a free market environment: Especially the interdependence of buyers and sellers. When a commodity is sold, sellers and buyers are linked in an immediate way since the price received by the first is disbursed by the latter. (Triffin 1947b) Interdependence between buyers and sellers might arise a bilateral monopoly, which in turn would imply that free markets automatically cause monopolies, which would mean that even in a free markets environment, entropy could not be maximized.

But according to Mises (1963) "monopoly prices emerge as differentiated from competitive prices" could only evolve if the demand for the product is inelastic. Hence, it is not "monopoly" as such that is catallactically relevant for Mises, but only the "configuration" of the demand function and the emergence of monopoly prices. (Mises.org 2005)

Thus, it is important to take a look on the demand side, meaning, if demand stays elastic, entropy can still be maximized even under monopolistic market conditions.

Monopolies don't tend to exist for a long time if exposed to a free market environment, even if entry-levels are high. Events like technology disruptions or evolutionary change in society and methods always threaten monopoly powers as they evolve out of nowhere and might be able to satisfy demand which was not even there before. Furthermore, it is important to remark that industries which have a higher rate of monopoly formation and longer persistence of monopoly power are those industries in which government has a large hand in regulation.

One may argue that monopolies don't even exist if there is no intervention into the market over a certain period of time, as there always exists a competitor in the market. It may only look like a monopoly only under myopically static analysis. Any broader definition of an industry exhibits an increased level of competition. Just narrow enough definitions of brand name products appear to have monopoly characteristics. In a competitive environment and without any governmental intervention, a company would not be able to set a price at a higher level and maintain it for a long period of time since consumers would create demand for products with prices well below the monopoly price. Thus, any realized dead weight loss by a monopoly set price would not prevail and increase entropy over time at the rate (17).

The same holds for subsidized monopolies. Since subsidies are a realization of a conditional probability of attaining a certain distribution, based on available 
market data at a time $t$, they realize and maintain the dead weight loss by not allowing entropy to increase. Due to the limitation of incorporated market information, entropy is capped at a certain level.

In a free market, subsidized monopolies would not exists since there would not exist any kind of subsidy. The subsidy is nothing else than the realization of the dead weight loss, caused by conditional redistribution. It is interesting to note that even subsidized monopolies are challenged by competition. A good example is given by the digital revolution which challenges even subsidized monopolies such as the electricity sector, e.g. by the evolvement of the blockchain technology and the shift towards de-centralized payment systems and residential prosumers. By jumps in technology, surge in innovation and changes in the social mindset it gets hard for subsidized monopolies to justify their subsidy. This shows again that, even if a company appears to be a monopoly, it won't be able to maintain its status as such over a longer period of time without governmental intervention. Exposure to a free market will have a disruptive effect to any monopoly as entropy maximization is the natural process, which breaks up any monopoly structures over time.

\section{Conclusion}

This article stresses the effect of wealth collection and distribution of allocable resources in a social market economy setting in contrast to a free market system. If no constraints are imposed on a system such as a free market economy, it will converge to an equilibrium at which all resources are optimally allocated. This equilibrium can only be realized if the entropy of the system is maximized, meaning that all offered and asked prices as well as transaction prices and quantities subject to the exchange are known and therefore contribute to a maximum complexity of the system.

The maximization of the entropy of a system leads to a convergence of all possible allocation distributions to an optimal distribution which is allocative fully efficient. The convergence process is characterized by an information increase of size $\mathrm{N}$, as well as an $\mathrm{N}$-trial learning process of the system. By identification of the level of entropy, the agents in the system learn in an iterative process how to allocate all available resources such that the entropy of the system is maximized. By maximizing the entropy, the agents automatically maximize the allocative efficiency of the system, which is Pareto-optimal.

In contrast to a free markets setup, any pre-determination of resource allocation, as done in a social market economy, restricts the information generation to let $N \rightarrow \infty$, and thus to maximize the system's entropy. The probability that this specific choice of distribution is maximizing the entropy of the system converges to zero when compared to the multiple adaption process of a free market system which converges to an optimal distribution with certainty.

The Kullback-Leibler divergence allows to measure the distance of a predetermined allocation from its optimal one, even if the optimal distribution is unknown. The relative distance to its optimum is compared to the relative distance of free market setup. The goal is to minimize the BIC information criterion, thus 
minimizing the KL-divergence. Minimizing the KL-divergence means maximizing the systems entropy in terms of its complexity. This can only be achieved by increasing information, which in turn leads to the maximization of entropy.

The probability that imposed taxes on resources for allocating them according to a pre-determined distribution is optimal, converges to zero, compared to the free market setup where information is generated and used to minimize the entropy.

Further, it is shown that the rate of social welfare loss from mis-allocated resources, caused by imposed taxes, equals the rate of accumulating the deadweight loss which occurs in a distorted economy.

The result implies that levying taxes reduces social welfare. This means that the realization of an inefficient allocation of resources leads to a lower level of living standard.

\section{References}

Ayres RU (1996) Eco-Thermodynamics: Economics and the Second Law: 2-3, Fontainebleau, France: NSEAD's Centre for the Management of Environmental Resources.

Boltzmann L (1866) Über die Mechanische Bedeutung des Zweiten Hauptsatzes der Wärmetheorie. [On the mechanic importance of the Second Fundamental Law of thermal theory]. Wiener Berichte, 53, 195-220, Volume I, paper 2, Reissued. New York: Chelsea 1969.

Case KE, Fair RC (1999) Principles of Economics (5th ed). Prentice Hall; Subsequent edition. ISBN 0-13-961905-4.

Frank RH (2003) Microeconomics and Behaviour (5 ${ }^{\text {th }}$ ed). NY: MacGraw Hill.

Gabriel B (2018) How Was the United States Government Funded Prior to Income Tax? Retrieved from DOI=https://pocketsense.com/united-states-government-funded-pri or-income-tax-12769.html.

Gibbs JW (1878) On the Equilibrium of Heterogeneous Substances. Transactions F, The Connecticut Academy Of Arts And Sciences, Vol. 3. New Haven: Tuttle, Morehouse \& Taylor, Printers.

Hausman J (1981) Exact consumer's surplus and deadweight Loss. American Economic Review 71(4): 662-76.

Hines JR (1999) Three sides of harberger triangles. Journal of Economic Perspectives 13(2): 167-188.

Jaynes ET (1965) Gibbs vs Boltzmann entropies. American Journal of Physics 33(5): 391398.

Kirzner IM (1973) Competition and Entrepreneurship. Chicago: University of Chicago Press, 20.

Lind H, Granqvist R (2010) A note on the concept of excess burden. Economic Analysis and Policy, 40(1): 63-73.

Maxwell JC (1871) Theory of Heat (reprinted 2001), ISBN 0-486-41735-2. New York: Dover.

Mises LV (1963) Human Action, USA: Yale University Press, 358-375. 
Mises.org (2005) A Critique of Neoclassical and Austrian Monopoly Theory. Mises Daily Articles. Retrieved from https://mises.org/library/critique-neoclassical-and-austrianmonopoly-theory.

Oliveira ARE (2014) 'The Fundamental Principles of Equilibrium and Motion' of Lazare Carnot. In: A History of the Work Concept. History of Mechanism and Machine Science, Vol 24. Springer, Dordrecht.

Robinson J (1969) Monopoly Equilibrium. The Economics of Imperfect Competition. London: Palgrave Macmillan. ISBN 978-1-349-15320-6.

Rothbard MN (1962) Man, Economy, and State with Power and Market. Ludwig von Mises Institute, Volume II. ISBN: 978-1-933550-27-5, 564.

Smith A (1776) The Wealth of Nations. Modern Library, Sixth Printing edition, Cannan E (January 25, 1994). ISBN-10: 0679424733.

Triffin R (1947a) Monopolistic Competition and General Equilibrium Theory, 58. UK: Cambridge Harvard University Press.

Triffin R (1947b) Monopolistic Competition and General Equilibrium Theory, 124. UK: Cambridge Harvard University Press.

Triffin R (1947c) Monopolistic Competition and General Equilibrium Theory, 133. UK: Cambridge Harvard University Press. 
